\title{
Cooperação entre Estado e iniciativa privada na difusão do carro elétrico: a futura construção de uma fábrica de carros elétricos no Rio de Janeiro
}

\author{
Claudia do Nascimento Martins ${ }^{1}$
}

\begin{abstract}
RESUMO
A futura construção de carros elétricos em Resende, no Estado do Rio de Janeiro, vem confirmar a importância da parceria, cooperação entre os agentes envolvidos na produção de um determinado produto para efetivar o seu desenvolvimento e a sua difusão. Como têm custos altos de produção, os carros elétricos precisam de incentivos governamentais para que sejam economicamente viáveis. Uma série de investimentos complementares deve ser realizada ao longo de sua cadeia produtiva, novos componentes deverão ser projetados e um novo conceito de postos de abastecimento deverá ser implementado. Assim, a parceria do Estado com os agentes privados, no caso a parceria com as empresas privadas que estão investindo na produção dos carros elétricos, é fundamental para o seu desenvolvimento. A difusão dos carros elétricos tende a beneficiar agentes econômicos direta e indiretamente envolvidos, pois gera ganhos expressivos para a indústria automobilística, indústria nascente de baterias de tração, de postos de recarga e também o setor elétrico.
\end{abstract}

Palavras-chave: carros elétricos; parceria; investimentos complementares; desenvolvimento; difusão

\begin{abstract}
The future construction of electric cars in Resende, in the state of Rio de Janeiro, confirms the importance of partnership and cooperation between the agents involved in the production of a particular product in order to accomplish its development and diffusion. Once they have higher costs of production, electric cars need government incentives for economic viability. A series of complementary investments should be made throughout the production chain, new components should be designed and a new concept of service stations should be implemented. Thus, the partnership between the State and private actors, in this case, the partnership with private companies that are investing in the production of electric cars, is fundamental to its development. The diffusion of electric cars tends to benefit economic agents involved directly and indirectly, since it generates significant gains for the automotive industry, the infant industry of traction batteries, the charging stations and also the electricity sector.
\end{abstract}

Keywords: electric cars; partnership; complementary investments; development; diffusion

\footnotetext{
${ }^{1}$ Economista e doutoranda em Políticas Públicas, Estratégias e Desenvolvimento (PPED), Instituto de Economia (IE), Universidade Federal do Rio de Janeiro (UFRJ). Professora da Universidade Veiga de Almeida (UVA). Membro da ABVE (Associação Brasileira de Veículo Elétrico). cnmartins@globo.com
} 


\section{Introdução}

$\mathrm{O}$ desenvolvimento significativo da indústria automobilística no século $\mathrm{XX}$ veio acompanhado de crescentes preocupações com o congestionamento de trânsito e a poluição gerada pelos carros que utilizam motor a combustão. Segundo Kemp e Soete (1990), no final do século XIX Londres já enfrentava enormes congestionamentos e também problemas ambientais, como resultado do uso de cavalos como meio de transporte. Estima-se que os cavalos não produzissem menos que $16 \mathrm{~kg}$ de excrementos por dia. A maioria das ruas de Londres era ocupada por cerca de 6.000 varredores, cuja tarefa não era manter as ruas limpas, mas apenas limpar o caminho para pedestres.

Meios alternativos de transporte estavam disponíveis há anos, mas não foram utilizados por causa de regulamentos restritivos. As empresas de transporte a cavalo, temendo a concorrência, conseguiram que o Parlamento da Inglaterra aprovasse a Lei da Bandeira Vermelha ${ }^{2}$ limitando a velocidade do veículo a vapor. Além disso, o veículo somente poderia trafegar precedido por um lampião, para prevenir acidentes, limitando-se assim a grande vantagem do vapor sobre os cavalos que era a velocidade.

Em relação aos cavalos, Kemp e Soete (1990) argumentam que os carros tinham, na época, um nível de cerca de 200 vezes menos emissões medido em gramas por milha, e que a passagem para os carros movidos por um motor, como um meio de transporte, tornou-se naquele momento um enorme alívio ambiental. Entretanto o problema é cumulativo e, após mais de um século, com milhões de automóveis com motor a combustão, o problema ambiental retornou.

O motor elétrico não é algo novo. Sua existência data do século XIX, quando os primeiros carros movidos a eletricidade foram projetados e construídos. No início do século $\mathrm{XX}$ os carros elétricos eram mais difundidos do que os carros movidos por motores a vapor e por motores a combustão. Entretanto, a crescente disponibilidade de postos de abastecimento como também o baixo custo de derivados de petróleo, associado à ausência de pressões ambientais, favoreceram a adoção do motor a combustão interna.

\footnotetext{
2 "O governo britânico decreta o Locomotives on Highways Act (Ato de Locomotivas em Estradas), que se torna mais conhecido como Lei da Bandeira Vermelha, de 1865. Ele ordena que todos os veículos rodoviários mecanicamente impelidos tenham velocidade limitada a 4 milhas por hora nas estradas abertas, e duas milhas por hora nas cidades, e que sejam precedidos sempre por um homem a pé, 50 jardas (cerca de 45 metros) à frente, brandindo uma bandeira vermelha de dia, e uma lanterna da mesma cor à noite, para avisar o público. A lei acaba com as locomotivas rodoviárias, a favor dos interesses ferroviários e dos negócios com veículos tracionados a cavalo" (Vieira, 2008:126).
} 
Os veículos automotivos vêm contribuindo para a contínua deterioração da qualidade do ar, especialmente nos grandes centros urbanos. Os veículos movidos a gasolina e a diesel produzem gases que constituem riscos graves tanto à saúde das pessoas quanto ao meio ambiente. Logo, nos últimos anos, com o notório aquecimento global causado pelas emissões de gases de efeito estufa, aumentou a busca por redução das emissões poluentes dos veículos como um todo, estimulando a investigação de novas tecnologias para a propulsão veicular.

Com o início da instabilidade no mercado mundial de petróleo e as discussões sobre o aquecimento global, uma janela de oportunidades se abre para o desenvolvimento tecnológico de bens e serviços que sejam poupadores de energia e contribuam para o desaquecimento global. Desta forma, a eletrificação veicular surge, no início do século XXI, como uma tendência tecnológica desejável, fazendo com que o carro elétrico volte ao cenário tecnológico mundial.

Trata-se de uma tecnologia que, embora não seja nova, não tem ainda uma trajetória tecnológica definida, mas tem um grande potencial de desenvolvimento em função de questões associadas aos impactos sobre o meio ambiente dos carros convencionais, ao custo crescente dos combustíveis derivados de fontes fósseis e à mobilidade urbana mais inteligente. Entretanto, parte-se do pressuposto de que o carro elétrico apresenta viabilidade econômica, desde que tenha um suporte e apoio de políticas públicas a fim de garantir as bases preliminares para a consolidação de um arranjo produtivo.

A futura construção de uma fábrica de carros elétricos da Aliança Renault-Nissan em Resende, no Estado do Rio de Janeiro, vem corroborar a importância da cooperação entre os agentes envolvidos na produção de um determinado bem com alta densidade tecnológica para fomentar o desenvolvimento tanto de sua cadeia produtiva como de sua difusão. Segundo Rogers e Schoemaker (1971), a difusão pode ser definida como o processo pelo qual uma inovação é transmitida por meio de determinados canais, ao longo do tempo, entre os membros de um sistema social. Assim, de acordo com Tigre (2006:71), "uma inovação só produz impactos econômicos abrangentes quando se difunde amplamente entre empresas, setores e regiões, desencadeando novos empreendimentos e criando novos mercados". A difusão nutre e orienta a trajetória da inovação, mostrando as necessidades da demanda por soluções técnicas.

Nesse contexto, o artigo tem como objetivo evidenciar a importância estratégica da cooperação entre Estado e agentes privados para a difusão do carro elétrico situando o projeto de construção de uma fábrica de carros elétricos no Estado do Rio de Janeiro. 


\section{O comportamento cooperativo: um breve argumento teórico}

Do ponto de vista da economia industrial, o setor automobilístico pode ser considerado um arranjo institucional. Segundo Fiani (2011:4), “os arranjos institucionais definem, por conseguinte, a forma particular como um sistema econômico coordena um conjunto específico de atividades econômicas". Assim, os arranjos institucionais tendem a definir a forma como as unidades econômicas podem cooperar como também competir. $\mathrm{O}$ desenvolvimento dos carros elétricos passa, de forma efetiva, por uma questão de coordenação desta atividade econômica, não sendo possível promover o desenvolvimento e a difusão dos carros elétricos sem que haja uma cooperação dos agentes envolvidos. Não havendo cooperação não há a possibilidade de alcançar o propósito de promover o desenvolvimento da sua cadeia produtiva, dado que o progresso tecnológico necessita de uma série de mudanças e investimentos que alteram significativamente as atividades econômicas.

Movimentos em direção à cooperação estão sendo realizados em muitos países. Empresas privadas em parceria com governos de diferentes países têm incentivado projetos e programas de $\mathrm{P} \& \mathrm{D}$ para a fabricação tanto dos ativos complementares que envolvem o carro elétrico - como as baterias - como o carro em si. Os ativos complementares representam um agregado que formam o conjunto de uma tecnologia ou produto, ou seja, uma tecnologia não cumpre seu papel de forma isolada e necessita de um conjunto de ativos complementares para funcionar. De acordo com Teece (1986), inovações tecnológicas requerem o uso de determinados ativos para produzir e distribuir novos produtos e serviços, ou seja, requerem o uso de um conjunto de outras capacidades ou ativos que são complementares.

A discussão estabelecida é de que os ativos complementares que foram surgindo para dar suporte ao motor a combustão, como grandes empresas de petróleo e postos de abastecimento, proporcionaram path dependence, ou seja, dependência de trajetória passada, indicando que o paradigma atual do motor a gasolina se manteve devido às ações ocorridas no passado (Cowan e Hultén, 1996). Assim, ativos complementares deverão ser desenvolvidos para difundir o carro elétrico. Caracterizam-se como ativos complementares do carro elétrico o desenvolvimento tecnológico de componentes, em particular as baterias, infraestrutura de carregamento que inclui equipamentos muito específicos e tipos de postos de carregamento.

Os desafios para a introdução dos carros elétricos no mercado mundial são enormes. Novos componentes deverão ser projetados, um novo conceito de postos de abastecimento 
deverá ser implantado, e a infraestrutura de energia elétrica deverá ser adaptada e possivelmente expandida. Neste sentido, pode-se prever a necessidade de definição e implementação de um novo conjunto de normas econômicas e sociais que deverão ser desenvolvidas.

De acordo com Fiani (2011:141), “as normas (econômicas) sociais... têm a capacidade de estabelecer e garantir regras que favoreçam as mudanças necessárias ao desenvolvimento, ao minimizarem as possibilidades de atuação oportunista e com isso reduzirem os custos de transação". No caso dos carros elétricos uma série de investimentos complementares deve ser realizada ao longo de sua cadeia produtiva. Entretanto, no momento da realização desses investimentos, se os agentes envolvidos começarem a atuar de forma oportunista, tentando obter condições mais vantajosas, o processo de desenvolvimento dos carros movidos à energia elétrica pode ser comprometido, ou até mesmo falhar. Assim, as normas sociais evitam o comportamento oportunista favorecendo então o comportamento cooperativo.

Como em toda tecnologia inovadora, mecanismos de incentivos e de fomento terão que ser implementados no favorecimento dos carros elétricos. O setor automobilístico, enquanto arranjo institucional, necessita claramente de apoio governamental para tornar viável um produto com tecnologia não convencional, mas que tem de ser compreendido mediante mudança no ambiente institucional inclusive por conta de questões ambientais e de mobilidade urbana. Davis e North (1971:6) definem ambiente institucional como "o conjunto de regras fundamentais de natureza política, social e legal, que estabelece a base para a produção, a troca e a distribuição".

\section{Cooperação entre os agentes para a difusão do carro elétrico}

Como em toda tecnologia inovadora, mecanismos de incentivos e de fomento deverão ser implementados em prol dos carros elétricos. Assim, uma nova indústria automobilística está a caminho e a cooperação entre os agentes envolvidos, principalmente o Estado, é fundamental para o desenvolvimento e difusão dos carros elétricos.

\subsection{A importância do Estado para o desenvolvimento do carro elétrico}

A importância do Estado na formação do novo paradigma tecnológico - os carros elétricos - é primordial. Um estado desenvolvimentista - o qual, no entendimento de Evans (2004), é aquele que consegue combinar autonomia e parceria - torna-se necessário para 
alavancar os carros movidos a energia elétrica. Segundo Rueschemeyer e Evans (1985:49), o "Estado deve adquirir certo grau de autonomia relativa da classe dominante com o objetivo de promover efetivamente a transformação econômica, sendo que essa autonomia relativa seria necessária não apenas para formular objetivos coletivos, mas para implementá-los também".

Rueschemeyer e Evans (1985) reconhecem que a tensão entre agir de acordo com o interesse geral da sociedade e como instrumento de dominação de grupos mais poderosos é intrínseca ao funcionamento do Estado. Desta forma, faz parte do Estado ser, simultaneamente, agente do bem comum e promotor de interesses particulares. Como assinalam, “... deve-se reconhecer que a ação do Estado, em apoio às tarefas fundamentais, muito provavelmente também tem implicações localizadas" (Rueschemeyer e Evans, 1985:48). De fato, quando o Estado promove o bem comum, que são os objetivos mais gerais da sociedade, as suas ações têm, frequentemente, resultados que são apropriados como benefícios por agentes privados. Assim, a difusão dos carros elétricos tende a beneficiar agentes econômicos direta e indiretamente envolvidos, pois gera ganhos expressivos para a indústria automobilística, indústria nascente de baterias de tração, de postos de recarga e também o setor elétrico.

Segundo Evans (2004:38), “somente quando há uma combinação entre a incorporação de interesses coletivos e a autonomia, um Estado pode ser chamado de desenvolvimentista. São imprescindíveis tanto autonomia como parceira". E ainda assinala que "a parceria, da forma aqui usada, implica um grupo concreto de conexões que ligam o Estado íntima e agressivamente a grupos sociais particulares com os quais o Estado compartilha projetos conjuntos de transformação" (Evans, 2004:93).

\subsection{Alguns exemplos de cooperação}

Os carros $100 \%$ elétricos têm apresentado evolução, mas, de forma concreta, terão que superar grandes obstáculos antes de se tornarem comuns no uso diário. Sua autonomia ainda é pequena e, em um país com grande extensão territorial, torna-se um grande problema, já que necessitaria de um complexo sistema de postos de recarga. Além disso, o tempo necessário para recarga é longo e os pacotes de baterias ainda são muito caros. Assim, o carro elétrico por apresentar um alto custo de produção tem necessitado de incentivos governamentais para que seja economicamente viável. Os governos de países como Dinamarca, Reino Unido, França, Estados Unidos, Holanda e Alemanha estão investindo em P\&D de baterias e criando incentivos para a aquisição dos carros elétricos através da redução da carga fiscal. Com a 
ausência de incentivos fiscais no Brasil, um carro elétrico, como o Leaf da Nissan, é vendido em torno de R \$ 200 mil; enquanto este mesmo carro é vendido nos Estados Unidos na ordem de R\$ 70 mil.

Entretanto, os incentivos fiscais para a aquisição do carro elétrico, apesar de importantes, não são suficientes. É necessário que se construa uma infraestrutura de abastecimento que com certeza provocará a difusão do veículo e Portugal tem sido um exemplo nessa questão. Em 2009, o governo português anunciou a implementação do Programa para a Mobilidade Elétrica em Portugal - o Mobi.E, que tem como principais objetivos a criação tanto de uma rede de carregamento como um modelo de serviço para o carro elétrico. O Programa Mobi.E, centrado no usuário, visa uma rede de carregamento acessível em qualquer ponto de Portugal e compatível com todas as marcas de veículos e aberta a todos os operadores (Adene, 2013).

A rede Mobi.E tem como metas fornecer bases para a difusão do veículo eléctrico, nas suas mais variadas vertentes e tipologias; projetar o país como estudo de caso internacional, promovendo a liderança de Portugal em soluções de carregamento de veículos elétricos; além de proporcionar, em parceria entre empresas e centros de excelência, condições para gerar e atrair investimentos para o desenvolvimento de soluções para a mobilidade elétrica (Adene, 2013).

$\mathrm{Na}$ Holanda a Fastned firmou parceria com a ABB, o grupo líder em tecnologias de eletricidade e automação, para fornecer a maior rede de carregamento rápido do país. Serão cerca de 200 postos espalhados pelas estradas do país, com capacidade para reabastecer um veículo em 15 a 30 minutos. A construção dos pontos de abastecimento deve ser concluída até 2015. Os equipamentos de recarga serão fornecidos pela $\mathrm{ABB}$ e cada carregador terá uma série de recursos de conectividade, como assistência remota, gerenciamento, manutenção e atualizações inteligentes de software (Automotive Business, 2013).

O plano para instalar postos de carregamento rápido de veículos elétricos nas autoestradas holandesas iniciou-se em 2011, quando a Fastned pediu autorização ao Ministério de Infraestrutura para instalar uma rede de carregamento de veículos elétricos. Em dezembro de 2011, o governo holandês anunciou o lançamento de um processo de licitação para fornecer de instalações de carregamento para as 245 estações de serviço existentes nas suas autoestradas. A Fastned, que ganhou a concessão para 201 estações, é uma empresa holandesa fundada em 2011 por Bart Lubbers e Michiel Langezaal com o objetivo de gerar uma infraestrutura de carregamento de âmbito nacional. A lógica do negócio baseia-se no 
conceito de que o primeiro a entrar nesse mercado poderá escolher as melhores localizações para o carregamento rápido no país, o que justifica um investimento precoce. Enquanto que a $\mathrm{ABB}$, sua parceira no projeto, é líder em tecnologias de energia e automação, possibilitando às empresas de eletricidade, água e gás e à indústria melhorar o seu desempenho, reduzindo o impacto ambiental. O Grupo ABB opera em cerca de 100 países e emprega aproximadamente 145.000 pessoas $(\mathrm{ABB}, 2013)$.

No Brasil, a cidade de Campinas, no interior de São Paulo, deve ser a primeira do país a contar com infraestrutura para receber os carros elétricos. O Programa de Mobilidade Elétrica é desenvolvido pela geradora e distribuidora CPFL Energia, empresa privada que deverá implantar uma rede de 100 pontos de eletricidade para o abastecimento desse tipo de veículo na cidade. Os carros serão importados da Europa e fornecidos pela Renault-Nissan. O projeto prevê a participação da prefeitura, do governo estadual, de agências reguladoras de energia e de várias empresas locais, que irão adquirir os veículos e incorporá-los às suas frotas, para uso de técnicos e engenheiros, que ficarão encarregados de fazer a avaliação da operação do sistema e analisar os impactos sobre a rede de distribuição de energia da cidade. O contrato de parceria prevê o fornecimento e a comercialização de um lote inicial de 50 veículos elétricos para as empresas participantes do pool (Planeta Sustentável, 2013).

O projeto na cidade de Campinas teve início em agosto de 2013, com a chegada dos três primeiros veículos - Zoe, Fluence e Kangoo, todos Z.E., sigla em inglês que significa emissão zero e, em seguida iniciaram-se testes na CPFL. O Zoe é o projeto de carro elétrico mais recente e mais eficiente da Renault, com autonomia para percorrer 210 quilômetros e um custo de carregamento das baterias de íon-lítio entre 7 e 8 reais. Na França, com todos os incentivos oficiais, o modelo custa em torno de 22 mil euros (o equivalente a 68 mil reais), enquanto que no Brasil deverá alcançar, com a incidência de todos os impostos, cerca de 215 mil reais (Exame Info, 2013).

Até o fim de 2013, mais seis veículos terão sido incorporados à frota da CPFL, para serem testados por funcionários da companhia. Em 2014, o programa será ampliado, envolvendo a participação de outras empresas, e feita a implantação da infraestrutura. A fase inicial do programa receberá 6,5 milhões de reais em investimentos entre veículos e infraestrutura. Até o final do programa esses investimentos podem alcançar R $\$ 25$ milhões (Jornal da Energia, 2013).

A ação da Renault-Nissan acontece em um momento em que os principais fabricantes instalados no Brasil, negociam, por meio da Anfavea, uma série de benefícios para a 
importação de veículos elétricos. As montadoras reivindicam que uma parcela da quota de importados, liberada para empresas enquadradas no Programa Inovar-Auto ${ }^{3}$ de 2013 do governo federal, seja preenchida por carros híbridos e elétricos, como também almejam isenção ou imposto reduzido para a importação de componentes, para o desenvolvimento de autopeças, visando a futura nacionalização ou a produção desses veículos nos próximos anos no Brasil.

\section{A implantação de um fábrica de carros elétricos no Estado do Rio de Janeiro}

A assinatura de um Memorando de Entendimentos, em junho de 2013, entre o governo do Estado do Rio de Janeiro e líderes dos setores público e privado representa um passo decisivo para a implantação de um fábrica de carros elétricos em Resende no Estado do Rio de Janeiro. A cidade fluminense foi escolhida para a construção da fábrica por sua proximidade com os portos de alta qualidade de Itaguaí e do Rio de Janeiro, disponibilidade para início da produção em curto espaço de tempo e fácil acesso à mão de obra qualificada e de fornecedores.

A unidade industrial deverá ser instalada pela Aliança Renault-Nissan e conta tanto com a ação conjunta das Secretarias de Transportes, de Desenvolvimento Econômico, Energia, Indústria e Serviços do Estado do Rio de Janeiro como também de empresas parceiras e comprometidas no projeto do carro elétrico - Light e Ampla que são principais distribuidoras de energia elétrica do estado, Petrobrás Distribuidora e Rio Negócios (Governo do Rio de Janeiro, 2013).

A Petrobras Distribuidora participa do projeto para estudar a implantação da infraestrutura para recarga dos carros elétricos em postos de serviços de bandeira Petrobras no Rio de Janeiro. A Light promoverá estudos para a implantação da infraestrutura necessária à rede de abastecimento/carregamento desses veículos. A Ampla irá estudar a implantação de infraestrutura tecnológica e inovadora, para garantir o suprimento de energia de forma sustentável. Enquanto que a Rio Negócios participará do grupo de trabalho de desenvolvimento do carro elétrico, representando a Prefeitura do Rio de Janeiro, com o propósito de promover comercialmente a cidade como destino de negócios desse setor

\footnotetext{
3 O Programa de Incentivo à Inovação Tecnológica e Adensamento da Cadeia Produtiva de Veículos Automotores (Inovar-Auto) é uma medida adotada pelo governo federal com o objetivo de estimular o investimento na indústria automobilística nacional.
} 
industrial e tecnológico emergente. A missão é identificar oportunidades e portfólios de projetos, desenvolver o polo setorial e consolidar a inteligência dessa nova atividade no país (Governo do Rio de Janeiro, 2013).

O grupo Renault-Nissan, na figura de seu CEO Carlos Ghosn, tenta revolucionar a indústria automobilística atraindo até mesmo seus concorrentes para o que é considerado o próximo passo do setor - o carro elétrico. A Renault-Nissan ao longo dos últimos anos tem realizado parcerias com prefeituras e estados de muitos países inserindo carros na forma de taxis ou mesmo participando de projetos de $\mathrm{P} \& \mathrm{D}$. O grupo é atualmente líder em vendas (61\%) de carros elétricos na Europa, com o maior portfólio de veículos do gênero em comercialização, e já investiu cerca de 4 bilhões de euros no desenvolvimento dessa tecnologia e produziu, entre dezembro de 2010 e julho de 2013, mais de 100 mil veículos movidos exclusivamente a eletricidade (Planeta Sustentável, 2013).

A futura construção de uma fábrica de carros elétricos em Resende é altamente relevante para o desenvolvimento econômico e tecnológico do Estado do Rio de Janeiro, bem como do país. Sua produção será primordial para alavancar um veículo de alta eficiência que tem como vantagens custos de utilização muito inferiores aos dos carros de motor a combustão como também níveis praticamente zero de emissões de gases poluentes.

Desta forma, para que a produção de carros elétricos seja em grande parte utilizada no próprio país, torna-se indispensável a instalação de pontos de recarga, tanto em locais com tempo maior de estacionamento, como também garagens residenciais e comerciais, e, principalmente, junto às vias públicas. Logo, com a fabricação dos carros elétricos no Brasil uma infraestrutura de abastecimento deverá surgir contribuindo, de fato, para a difusão. Consequentemente, a política de impostos para estes veículos deverá ser revista, em especial o IPI. Atualmente não há uma definição específica, logo os carros elétricos encontram-se na categoria "outros", cuja alíquota de $25 \%$ é inviável, desestimulando os possíveis compradores.

\section{Conclusão}

A indústria automobilística inicia o século XXI com a perspectiva de um novo paradigma tecnológico. Novos avanços ocorrem e deverão ocorrer no que tange aos carros totalmente elétricos e recarregá-los poderá ser mais natural do que parar para abastecê-los, como se faz atualmente. Contudo, é relevante que haja integração entre empresas privadas e 
governo para o desenvolvimento de ativos complementares que envolvam o carro elétrico, especialmente incentivos fiscais e infraestrutura de abastecimento.

A cooperação, parceria do Estado com os agentes privados, no caso com as empresas privadas que estão investindo na produção dos carros elétricos, é fundamental para promover o seu desenvolvimento. Embora países europeus e Estados Unidos disponibilizem incentivos fiscais para os carros elétricos, eles ainda demonstram pouca atratividade aos consumidores devido principalmente à insuficiente infraestrutura de carregamento. Nestes termos, os incentivos ao desenvolvimento industrial são relevantes, mas incluir a criação de uma infraestrutura é fundamental. O Estado em cooperação com os agentes privados deve criar condições efetivas para não somente o desenvolvimento tecnológico do carro movido a energia elétrica, como também a sua difusão.

Neste sentido, pode-se assumir que a consolidação do arranjo produtivo do carro elétrico, em função dos desafios e incertezas tecnológicas, depende de políticas públicas que integrem os agentes produtivos.

\section{Referências bibliográficas}

ABB. Fastened seleciona a ABB para construir a maior rede holandesa de carregamento rápido de veículos eléctricos. http://www.abb.com/cawp/seitp202/e68bb83ec1bc8fbbc1257bb40059727e.aspx. Acesso em 26 de outubro de 2013.

ADENE, Agência para energia. Mobi.E. Disponível em: http://www.adene.pt/ptpt/NavegacaoDeTopo/EnergiaNosTransportes/MobilidadeElectrica/Paginas/MobiE.aspx. Acesso em 26 de outubro de 2013.

AUTOMOTIVE BUSINESS. Fastned e ABB implementaram rede de recarga rápida na Holanda. Disponível em: http://www.automotivebusiness.com.br/noticia/17438/fastnede-abb-implementarao-rede-de-recarga-rapida-na-holanda. Acesso em 26 de outubro de 2013.

COWAN, R.; HULTÉN, S. Escape lock-in: the case of the electric vehicle. Technological Forecasting and Social Change, vol. 53, n. 1, pp. 61-80, 1996.

DAVIS, Lance; NORTH, Douglass C. Institutional change and American economic growth. Cambridge: Cambridge University Press, 1971. 
EVANS, Peter. Autonomia e parceria. Estados e transformação industrial. Rio de Janeiro: UFRJ, 2004.

EXAME INFO. Campinas (SP) vai implantar rede de 100 postos para carro elétrico.

Disponível em: http://info.abril.com.br/noticias/bitnocarro/2013/09/campinas-sp-vaiimplantar-rede-de-100-postos-para-carros-eletricos.shtml. Acesso em 29 de outubro de 2013.

FIANI, Ronaldo. Cooperação e conflito: instituições e desenvolvimento econômico. Rio de Janeiro: Elsevier, 2011.

GOVERNO DO RIO DE JANEIRO. Rio de janeiro lança parceria público-privada para transporte com emissão zero. Disponível em:

http://www.rj.gov.br/web/sedeis/exibeconteudo?article-id=1635917. Acesso em 29 de outubro de 2013.

JORNAL DA ENERGIA. CPFL Energia investirá até $R \$ 25$ milhões em estudos para eletropostos. Disponível em: http://www.jornaldaenergia.com.br/ler_noticia.php?id_noticia=14728\&id_secao=38. Acesso em 29 de outubro de 2013.

KEMP, René; SOETE, Luc. Inside the 'green box': on the economics of technological change and the environment. The economics of technical change and the environment. 1990. Disponível em: http://www.ie.ufrj.br/hpp/intranet/pdfs/inside_the_green_box.pdf. Acesso em 25 de outubro de 2013.

KEMP, René; ORSATO, Renato J.; DIJK, Marc. The emergence of an electric mobility trajectory. Energy Policy n. 52, 135-145, 2013.

PLANETA SUSTENTÁVEL. Campinas implantará rede de 100 postos elétricos. Disponível em: http://planetasustentavel.abril.com.br/noticias/campinas-implantara-rede-100postos-carros-eletricos-752416.shtml. Acesso em 29 de outubro de 2013.

ROGERS, E; SCHOEMAKER, F. Communication of Innovations: a Cross Cultural Approch. Free Press, 1971.

RUESCHEMEYER, Dietrich; EVANS, Peter B. The state and economic transformation: toward an analysis of the conditions underlying effective intervention. In: EVANS, Peter B.; RUESCHEMEYER, Dietrich; SKOCPOL, Theda. Bringing the state back in. Cambridge: Cambridge University Press, 1985. 
TEECE, D. J. Profiting from technological innovation, Research Policy, 15(6), pp. 285-305, 1986.

TIGRE, Paulo Bastos. Gestão da inovação: a economia da tecnologia no Brasil. Rio de Janeiro: Elsevier, 2006.

VIEIRA, José Luiz. A história do automóvel: a evolução da mobilidade. Volume 1. São Paulo: Alaúde Editorial, 2008. 